“ (C) 2013 IEEE. Personal use of this material is permitted. Permission from IEEE must be obtained for all other uses, in any current or future media, including

reprinting/republishing this material for advertising or promotional purposes, creating new collective works, for resale or redistribution to servers or lists, or reuse of any copyrighted component of this work in other works." 


\section{Generalized Local N-ary Patterns for Texture Classification}

\author{
Sheng Wang \\ University of Technology, Sydney \\ 15 Broadway, Ultimo, NSW 2007 \\ Sheng.Wang-1@student.uts.edu.au \\ Xiangjian $\mathrm{He}$ \\ University of Technology, Sydney \\ 15 Broadway, Ultimo, NSW 2007 \\ Xiangjian. He@uts.edu.au
}

\author{
Qiang Wu \\ University of Technology, Sydney \\ 15 Broadway, Ultimo, NSW 2007 \\ Qiang.Wu@uts.edu. au \\ Jie Yang \\ Shanghai Jiao Tong University \\ 800 DongChuan Rd., Shanghai 200240 \\ JieYang@sjtu.edu.cn
}

\begin{abstract}
Local Binary Pattern (LBP) has been well recognised and widely used in various texture analysis applications of computer vision and image processing. It integrates properties of texture structural and statistical texture analysis. LBP is invariant to monotonic gray-scale variations and has also extensions to rotation invariant texture analysis. In recent years, various improvements have been achieved based on LBP. One of extensive developments was replacing binary representation with ternary representation and proposed Local Ternary Pattern (LTP). This paper further generalises the local pattern representation by formulating it as a generalised weight problem of Bachet de Meziriac and proposes Local N-ary Pattern (LNP). The encouraging performance is achieved based on three benchmark datasets when compared with its predecessors.
\end{abstract}

\section{Introduction}

Local image patterns are very important in texture classification. The Local Binary Pattern (LBP), as one of the most widely used texture descriptors, has been successfully applied to many computer vision tasks, such as face recognition, texture recognition and shape localization [7][13][4][17]. LBP compares the intensity value of a center pixel to that of its neighborhood pixels and uses a binary thresholding function to extract binary patterns according to comparison results [10]. Following LBP, a large number of improved texture descriptors have been proposed. Those improvements are categorized into fusionbased methods, geometry-based methods, and arity-based methods.
Fusion-based improvement Fusion-based improvement integrates multple local pattern representations [18][8].

In [18], the Completed LBP (CLBP) is proposed. In order to enhance the discriminability of the descriptor, the orginal LBP is generalized to CLBP-Sign (CLBP_S) in [18]. In addition, CLBP-Magnitude (CLBP_M) and CLBP-Center (CLBP_C) are also proposed. The integration of CLBP_S, CLBP_M (magnitude of neighborhood pixels) and CLBP_C (gray-level of the center pixel) forms the CLBP map, which provides extra information to improve the LBP descriptor.

In [8], two novel descriptors, Elongated LTP (ELTP) and Improved LTP (ILTP) are proposed. The ELTP is obtained by replacing LBP in Elongated LBP (ELBP) [12] with LTP. The ELBP performs better when anisotropic structure exists in images. The ILTP is obtained by replacing LBP in Improved LBP (ILBP) [6] with LTP. The ILBP works better against the effect of noise. Both ELTP and ILTP outperform the LBP descriptor in [8].

Geometry-based improvement Geometry-based improvement alters binary comparison scheme in the original LBP [5][3].

In [5], the Center Symmetric LBP (CS-LBP) is proposed. In order to encode spatial information of the image, the CS-LBP first divides an image using a $4 \times 4$ grid, then process the image sub-block in each cell. Instead of comparing the intensity value of a center pixel to those of its neighborhood pixels, the CS-LBP compares the intensity value of each of those eight neighborhood pixels with that of its center-symmetric counterpart (a total of four comparisons). As a result, the CS-LBP only produces $2^{4}=16$ different binary patterns. Compared to LBP, the CS-LBP captures the gradient information better, hence the CS-LBP achieves significant reduction in dimensionality while preserving distinctiveness. 
In [3], in order to achieve more efficient texture classification, the Binary Gradient Contour (BGC) texture descriptor is proposed. There are three differnt types of BGC descriptors, the single-loop, the double-loop, and the tripleloop. The single-loop BGC descriptor generates local binary patterns by comparing the intensity value of each of those eight neighboring pixels with that of its immediate adjacent pixel following clockwise direction. More details are found in [3].

Arity-based improvement Arity-based descriptors rely on a sophisticate thresholding function and local patterns of higher arity to explore more discriminant features from the local texture [14].

The Local Tenary Pattern (LTP) is proposed to alleviate the sensitivity of LBP to the random and quantization noise in uniform and near-uniform image regions [14]. The LTP uses a ternary thresholding function and forms a ternary local pattern of $\{-1,0,1\}$. Such tenary local pattern is then split into a pair of binary patterns, termed as the upper pattern and the lower pattern, respectively. The upper pattern and the lower pattern are then concatenated together to form the LTP representation.

In this paper, we further propose the Local N-ary Pattern (LNP) in order to enhance the discriminability of the local pattern. LNP belongs to the Arity-based descriptors. However, different from LTP, we do not split the $\mathrm{N}$-ary pattern into $\mathrm{N}-1$ binary patterns, instead a $\mathrm{N}^{8}$-vector is formed. The reason for not splitting is explained in Section 3.1.

Our contributions are summarised as follows.

Firstly, we formulate local image representation as a weight problem of Bachet de Meziriac [2]. This formulation enables us to take advantage of the solution to the weight problem of Bachet de Meziriac to extract better image patterns.

Secondly, we propse a uniform framework to generalize the local pattern representation. By replacing the binary representation in LBP and the ternary representation in LTP with $\mathrm{N}$-ary representation, the Local $\mathrm{N}$-ary Pattern (LNP) is proposed. The LBP is in fact a special case of LNP (i.e. $N=2$ ). The LNP with $\mathrm{N}$ greater than two outperforms both LBP and LTP in our texture classification experiments.

The rest of this paper is organized as follows. In Section 2.3, the motivation of LNP-The Weight Problem of Bachet de Meziriac and its generalization is introduced. In Section 3.1, the LNP descriptor is proposed, an example comparison between LNP and LTP is also provided. Experimental results are detailed in Section 4. Section 5 concludes this paper and briefly mention future works.

\section{Related works}

\subsection{Local Binary Patterns}

LBP is one of the most widely used texture descriptor. The process for LBP feature extraction (using $3 \times 3$ patch size) is illustrated in Fig. 1.

In Fig. 1, the binary thresholding function is represented by

$$
a_{i}=\left\{\begin{array}{ll}
1 & \text { if } p_{i}>c \\
0 & \text { if } p_{i} \leq c
\end{array},(1 \leq i \leq 8) .\right.
$$

In (1), $c$ is the intensity value of the center pixel, and $p_{i}$ is the intensity value of the neigborhood pixels.

The weighted sum function is represented by

$$
V_{B}=\sum_{i=1}^{8} a_{i} \cdot 2^{i-1},\left(a_{i} \in\{0,1\}\right) .
$$

\subsection{Local Ternary Patterns}

As an extension of LBP, the LTP is more reliable because it uses a ternary thresholding function, which is more resistant to noise. The process for LTP feature extraction (using $3 \times 3$ patch size) is illustrated in Fig. 2 .

In Fig. 2, the ternary thresholding function is represented by

$$
a_{i}= \begin{cases}1, & \text { if } p_{i}>c+t \\ 0, & \text { if } c-t \leq p_{i} \leq c+t \quad, \quad(1 \leq i \leq 8) . \\ -1, & \text { if } p_{i}<c-t\end{cases}
$$

In (3), $c$ is the intensity value of the center pixel, $p_{i}$ is the intensity value of the neigborhood pixels, and $t$ is a threshold value that controls the sensitivity of the ternary pattern against noise.

The binary thresholding function for the upper pattern is represented by

$$
u_{i}=\left\{\begin{array}{ll}
1 & \text { if } a_{i}=1 \\
0 & \text { otherwise }
\end{array},(1 \leq i \leq 8) .\right.
$$

The binary thresholding function for the lower pattern is represented by

$$
l_{i}=\left\{\begin{array}{ll}
1 & \text { if } a_{i}=-1 \\
0 & \text { otherwise }
\end{array},(1 \leq i \leq 8) .\right.
$$

The weighted sum function for the upper pattern is represented by

$$
V_{u}=\sum_{i=1}^{8} u_{i} \cdot 2^{i-1},\left(u_{i} \in\{0,1\}\right) .
$$

The weighted sum function for the lower pattern is represented by

$$
V_{l}=\sum_{i=1}^{8} l_{i} \cdot 2^{i-1},\left(l_{i} \in\{0,1\}\right)
$$




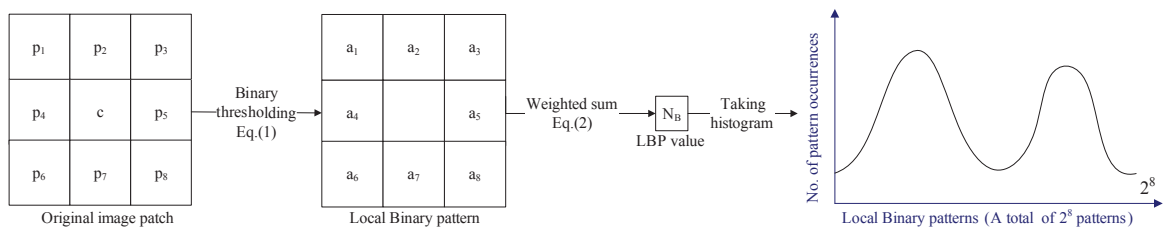

Figure 1: LBP feature extraction process

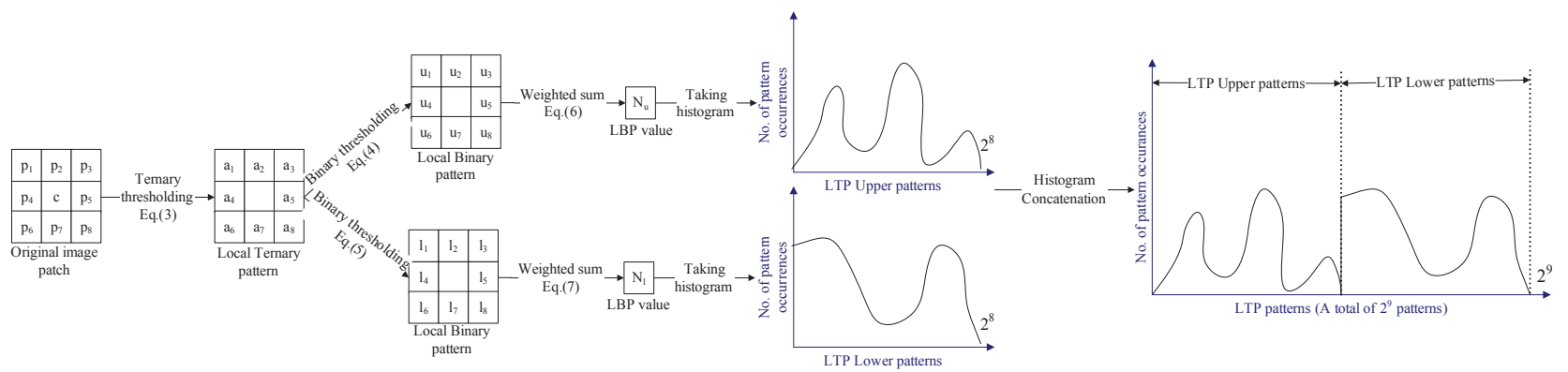

Figure 2: LTP feature extraction process

The histogram of the upper pattern and the histogram of the lower pattern are concatenated together to form the LTP representation, as illutrated in Fig. 2.

\subsection{Generalized Weight Problem of Bachet de Meziriac}

Motivated by the binary representation of LBP and the ternary representation of LTP, the local pattern representation is converted to The Weight Problem of Bachet de Meziriac [2], which is briefly introduced here. More details are found in [2].

The Weight Problem of Bachet de Meziriac A merchant had a forty pound weight that was broken into four pieces as a result of a fall. When the pieces were subsequently weighed, it was found that the weight of each piece was a whole number of pounds and that the four pieces could be used to weight every integer weight between one and forty pounds. What were the weights of the pieces? (Solution: The weights of those pieces are one pound, three pounds, nine pounds, and twenty-seven pounds.)

The solution to the problem implies that any arbitrary integer weight $W$ is expressed by a linear equation as follows

$$
W=\sum_{i=1}^{K} a_{i} \cdot 3^{i-1},\left(a_{i} \in\{-1,0,1\}\right) .
$$

The differences between (8) and (2) are the base of exponential expression and the range of the coefficients. In LBP, such exponential expressions are used to assign weights to different bits of the binary pattern such that the
LBP value is unique for each unique binary pattern. In the Weight Problem of Bachet de Meziriac, such exponential expressions correspond to those pieces of the broken weight. Any integer weight is balanced by a unique set of placement of those piece on the scale.

Without loss of generality, let $V$ be an arbitrary nonnegative integer, and let $b$ be an integer base of the exponential expression that is greater than one. If $b$ is increased, the Weight Problem of Bachet de Meziriac is further generalized through deduction approach. In our study, it is observed that if $a_{i} \in\{-1,0,1,2\}$ and $b=4$, all nonnegative integers are represented by linear equations such as (8). Equations such as (8) can also be used to represent the case when $a_{i} \in\{-2,-1,0,1,2\}$ and $b=5$.

Generally, $V$ is represented by

$$
V=\sum_{i=1}^{K} a_{i} \cdot b^{i-1}, \underset{(b>1 \wedge b \in Z)}{\left(a_{i} \in[f(b), g(b)] \wedge a_{i} \in Z\right)} .
$$

In (9), $f(b)$ is represented by

$f(b)=\left\{\begin{array}{ll}\frac{2-b}{2} & \text { if } b \text { is even } \\ \frac{1-b}{2} & \text { if } b \text { is odd }\end{array},(b>1 \wedge b \in Z)\right.$.

In (9), $g(b)$ is represented by

$g(b)=\left\{\begin{array}{ll}\frac{b}{2} & \text { if } b \text { is even } \\ \frac{b-1}{2} & \text { if } b \text { is odd }\end{array},(b>1 \wedge b \in Z)\right.$.

The vectorized form of (9) is

$$
\mathbf{V}=\mathbf{A B}
$$


If $b=4, \mathbf{V}, \mathbf{A}$, and $\mathbf{B}$ are

$\mathbf{V}=\left|\begin{array}{c}0 \\ 1 \\ 2 \\ 3 \\ 4 \\ 5 \\ 6 \\ 7 \\ 8 \\ \vdots \\ 42\end{array}\right| . \mathbf{A}=\left|\begin{array}{cccc}0 & 0 & 0 & \cdots \\ 1 & 0 & 0 & \cdots \\ 2 & 0 & 0 & \cdots \\ -1 & 1 & 0 & \cdots \\ 0 & 1 & 0 & \cdots \\ 1 & 1 & 0 & \cdots \\ 2 & 1 & 0 & \cdots \\ -1 & 2 & 0 & \cdots \\ 0 & 2 & 0 & \cdots \\ \vdots & \vdots & \vdots & \vdots \\ 2 & 2 & 2 & \cdots\end{array}\right| . \mathbf{B}=\left|\begin{array}{c}4^{0} \\ 4^{1} \\ 4^{2} \\ \vdots\end{array}\right|$.

Alternative representations are found when $b=5$.

\section{Proposed framework}

\subsection{The Local $\mathrm{N}$-ary Patterns}

As shown in Fig. 1, two functions are needed to convert an image patch to its unique integer representation. Those functions are the thresholding function and the weighted sum function.

In general, the $\mathrm{N}$-ary thresholding function $\phi$ is represented by

$$
\begin{cases}f(b), & \text { if } x \leq \Delta_{1} \\ f(b)+1, & \text { if } \Delta_{1}<x \leq \Delta_{2}, \cdots, \Delta_{|f(b)|+g(b)} \\ \cdots & \text { if } \Delta_{|f(b)|}<x \leq \Delta_{|f(b)|+1} \\ 0, & \text { if } \Delta_{|f(b)|+1}<x \leq \Delta_{|f(b)|+2} \\ 1, & \text { if } \Delta_{|f(b)|+g(b)-1}<x \leq \Delta_{|f(b)|+g(b)} \\ \cdots & \text { if } x>\Delta_{|f(b)|+g(b)}\end{cases}
$$

In (14), the definition of $f(b)$ is the same as in (10) and the definition of $g(b)$ is the same as in (11). Thresholds are determined in the way below.

When $\phi(x)=0$, the threshold value $\Delta_{|f(b)|}$ is represented by

$$
\Delta_{|f(b)|}=c-t .
$$

The threshold value $\Delta_{|f(b)|+1}$ is represented by

$$
\Delta_{|f(b)|+1}=\left\{\begin{array}{ll}
c, & \text { if } b \text { is even } \\
c+t, & \text { if } b \text { is odd }
\end{array} .\right.
$$

Threshold values less than $\Delta_{|f(b)|}$ are represented by

$$
\Delta_{i}=\Delta_{i+1}-t, \underset{(1 \leq i \leq|f(b)|-1)}{(i \in Z)} .
$$

Threshold values greater than $\Delta_{|f(b)|+1}$ are represented by

$$
\Delta_{j}=\Delta_{j-1}+t, \underset{(j \in Z)}{(j \in Z)|+2 \leq j \leq| f(b) \mid+g(b))} .
$$

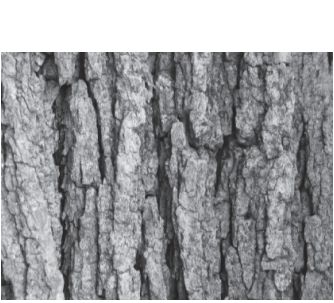

(a) Original texture image (b) Local patterns of (a), LBP in red, LTP in green, LNP $(\mathrm{N}=3)$ in blue

Figure 3: Illustration of original image and its LBP, LTP and LNP $(N=3)$ representations (Best viewed in color)

In (15), (16), (17) and (18), definitions of $c$ and $t$ are the same as that of (3).

Using the $\mathrm{N}$-ary thresholding function (14), N-ary patterns are extracted from images. Using the weighted sum function (9), those $\mathrm{N}$-ary patterns are converted to integers. By taking the histogram of those integer, the LNP representation is obtained. LBP is a special case of LNP with the base of the exponential expression $b=2$ ( $b$ is defined in (9)).

Fig. 3 illustrates the texture representation of LBP, LTP, and $\operatorname{LNP}(N=3)$ for an image.

In contrast to LTP, we do not split our LNP representation because it is capable of representing more discriminant information. As illustrated in the case study detailed in Fig. 4, although both LTP and LNP $(N=3)$ are represented by $\{-1,0,1\}$, the discriminality of LNP is better than that of the LTP.

\subsection{One-against-rest SVM}

Having obtained the LNP representation, the next step is to train an one-against-rest SVM classifier [1]. The SVM classifier is favored because of its good performance in classification accuracy. One-against-rest is one of the most widely used method for dealing with a multi-class classification problem, which is the case in our texture classification experiments. The optimal SVM paramters are determined according to the experimental results.

\section{Experimental results}

Three publicly available datasets are used in our experiments, Brodatz texture dataset, CUReT 92 gray images subset, and UIUC texture dataset [15]. On each dataset, four independent experiments were carried out. In each experiment, the mean classification accuracy over all texture categories are calculated. In each texture category, 20 samples are randomly selected for training and all the rest are used for testing. The average of mean classification accuracies on those four experiments are used to measure the 

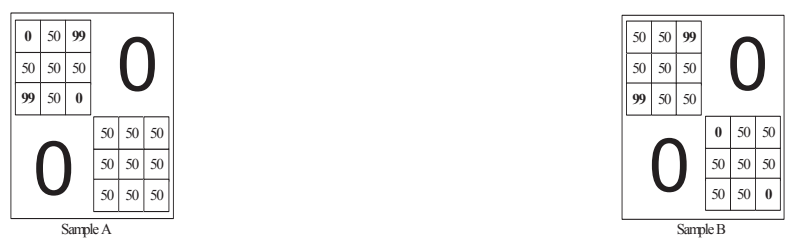

(a) Original texture for sample A and sample B (numbers represent gray-level intensity)
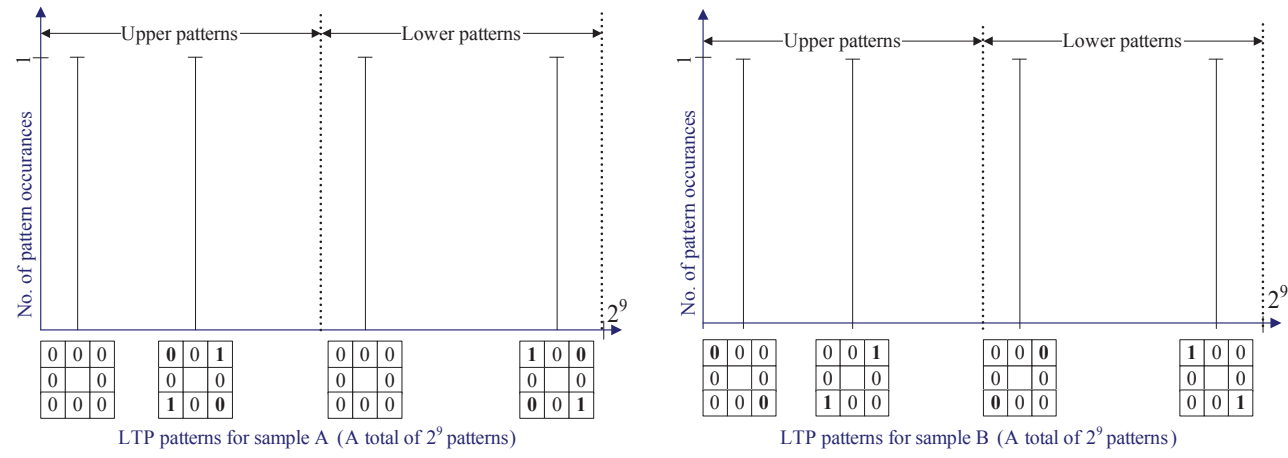

(b) LTP representation for sample A and sample B
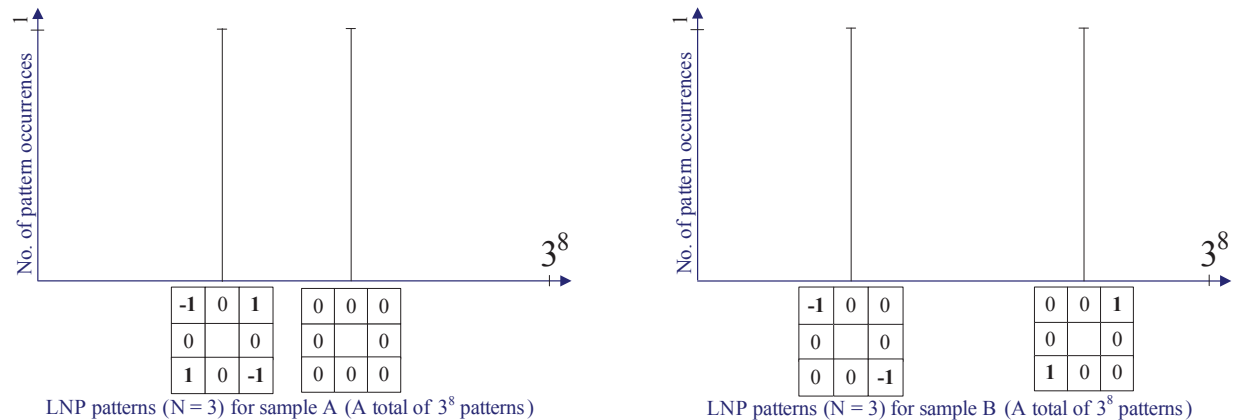

(c) $\mathrm{LNP}(\mathrm{N}=3)$ representation for sample $\mathrm{A}$ and sample $\mathrm{B}$

Figure 4: Comparison between LTP and LNP (when $N=3$ )

Table 1: Details of three datasets

\begin{tabular}{|l|c|c|c|}
\hline & Brodatz & CUReT gray & UIUC \\
\hline \# of images & 64 & 92 & 40 \\
\hline \# of classes & 32 & 61 & 25 \\
\hline image size & $64 \times 64$ & $200 \times 200$ & $640 \times 480$ \\
\hline
\end{tabular}

performance. Details for those three datasets are found in Table 1.In our experiments, the patch size is $3 \times 3$. The dimensionality of the LNP descriptor for $\mathrm{N}$ equals 3,4 , and 5 are 6561,65536 , and 390625 , respectively.

\subsection{Average of mean classification accuracy}

The mean classification accuracy is one of the most commonly used measurements in multi-class classification. The experimental results for the average of mean classification accuracies on all three datasets are illustrated in Table 2. In Table 2, it is found that the $\operatorname{LNP}$ (with $N=3$ )
Table 2: Average of mean classification accuracies (in percentage)

\begin{tabular}{|c|c|c|c|}
\hline & UIUC & CUReT gray & Brodatz \\
\hline LBP $^{*}$ & $75.95 \pm 0.44$ & $90.5 \pm 0.97$ & $86.47 \pm 0.42$ \\
\hline LTP & $84.45 \pm 1.55$ & $90.17 \pm 1.03$ & $94.35 \pm 0.75$ \\
\hline LNP(3) & $85.8 \pm 0.98$ & $91.0 \pm 0.55$ & $95.1 \pm 0.52$ \\
\hline LNP(4) & $\mathbf{8 7 . 9 5} \pm \mathbf{0 . 8 5}$ & $\mathbf{9 1 . 2 5} \pm \mathbf{0 . 8}$ & $\mathbf{9 5 . 2 1} \pm \mathbf{0 . 2 6}$ \\
\hline LNP(5) & $87.2 \pm 1.67$ & $87.79 \pm 0.9$ & $93.98 \pm 0.42$ \\
\hline
\end{tabular}

*The LBP implementation is provided by the author of [16].

outperforms both LBP and LTP in terms of average of mean classification accuracy, and optimal results are obtained when $N=4$. However, the performance drops as $\mathrm{N}$ further increases, we attribute this performance drop to the curse of dimensionality problem. 
Table 3: Optimal mean classification accuracy (in percentage) for different threshold values

\begin{tabular}{|c|c|c|c|}
\hline & UIUC & CUReT gray & Brodatz \\
\hline$t=5$ & $\mathbf{8 9 . 2}$ & 91.4 & 92.97 \\
\hline$t=10$ & 89 & $\mathbf{9 2 . 2 8}$ & 94.39 \\
\hline$t=15$ & 87.2 & 91.8 & 94.32 \\
\hline$t=20$ & 86.4 & 91.6 & 95.17 \\
\hline$t=25$ & 85.8 & 90.66 & 95.17 \\
\hline$t=30$ & 85.2 & 90.8 & 95.38 \\
\hline$t=35$ & 85.2 & 90.16 & 95.1 \\
\hline$t=40$ & 85.6 & 90.23 & $\mathbf{9 5 . 5 3}$ \\
\hline
\end{tabular}

In our experiments, it is also found that the optimal threshold values differ for different datasets. Such differences are illustrated in Table 3.

The reason that better classification accuracy is achieved on Brodatz dataset using larger threshold value is that texture images in Brodatz dataset have a wider range in intensity values compared to those of UIUC dataset and CUReT gray dataset. Therefore, using larger threshold values can better capture the distinctiveness of texture images in the Brodatz dataset.

\section{Conclusions and future works}

In conclusion, we propose the LNP framework for texture classification. The LNP is motivated by The Weight Problem of Bachet de Meziriac and its generalization. The LNP is higher in discriminability than both LBP and LTP. It has been proven in texture classification experiments that LNP outperforms both LBP and LTP, achieving higher mean classification accuracy.

The LNP representation can be further improved using dimensionality reduction and three methods can be considered, dimensionality reduction using discriminant analysis [9], dimensionality reduction using uniform pattern [16], and dimensionality reduction using sparse coding [11]. Using dimensionality reduction, computational costs can be reduced while the mean classification accuracy stays competitive.

\section{References}

[1] S. Canu, Y. Grandvalet, V. Guigue, and A. Rakotomamonjy. Svm and kernel methods matlab toolbox. Perception Systemes et Information, INSA de Rouen, Rouen, France, 2005.

[2] H. Dorrie. 100 great problems of elementary mathematics:their history and solution. 1965.

[3] A. Fernandez, M. X. Alvarez, and F. Bianconi. Image classification with binary gradient contours. Optics and Lasers in Engineering, 49(9-10):1177 - 1184, 2011.

[4] G.Zhao and M.Pietikainen. Dynamic texture recognition usng local binary patterns with an application to facial ex- pressions. IEEE Transactions on Pattern Anal. Mach. Intell., 27(6):915-928, 2007.

[5] M. Heikkil, M. Pietikinen, and C. Schmid. Description of interest regions with center-symmetric local binary patterns. In Computer Vision, Graphics and Image Processing, volume 4338, pages 58-69, 2006.

[6] H.Jin, Q.Liu, H.Lu, and X.Tong. Face detection using improved lbp under bayesian framework. In Proceedings of International Conference on Image and Graphics, pages 306309, 2004.

[7] D. Huang, C. Shan, M. Ardabilian, Y. Wang, and L. Chen. Local binary patterns and its application to facial image analysis: A survey. IEEE Transactions on Systems, Man, and Cybernetics, Part C: Applications and Reviews, 41(6):765-781, 2011.

[8] A. L.Nanni, S.Brahnam. A local approach based on a local binary patterns variant texture descriptor for classifying pain states. Expert Syst. Appl., 37(12):7888-7894, 2010.

[9] A. M. Martinez and A. Kak. Pca versus lda. Pattern Analysis and Machine Intelligence, IEEE Transactions on, 23(2):228-233, 2001.

[10] T. Ojala, M. Pietikainen, and D. Harwood. Performance evaluation of texture measures with classification based on kullback discrimination of distributions. In Proceedings of the 12th IAPR International Conference on Pattern Recognition, volume 1, pages 582-585, 1994.

[11] B. A. Olshausen and D. J. Fieldt. Sparse coding with an overcomplete basis set: a strategy employed by v1. Vision Research, 37:3311-3325, 1997.

[12] S.Liao and A. Chung. Face recognition by using elongated local binary patterns with average maximum distance gradient magnitude. In Proceedings of the Eigth Asian Conference on Computer Vision, pages 672-679, 2007.

[13] T.Ahonen, A.Hadid, and M.Pietikainen. Face recognition with local binary patterns: Application to face recognition. IEEE Transactions on Pattern Anal. Mach. Intell., 28(12):2037-2041, 2006.

[14] X. Tan and B. Triggs. Enhanced local texture feature sets for face recognition under difficult lighting conditions. In Analysis and Modeling of Faces and Gestures, volume 4778, pages 168-182, 2007.

[15] R. Timofte and L. V. Gool. A training-free classification framework for textures, writers, and materials. In Proceedings of the British Machine Vision Conference, pages 93.193.12. BMVA Press, 2012.

[16] T. T.Ojala, M.Pietikainen. Multiresolution gray-scale and rotation invariant texture classification with local binary patterns. IEEE Transactions on Pattern Analysis and Machine Intelligence, 24(7):971-987, 2002.

[17] X.Huang, S.Z.Li, and Y.Wang. Shape localization based on statistical method using extended local binary pattern. In Proceedings of International Conference on Image and Graphics, pages 184-187, 2004.

[18] Z.Guo, L.Zhang, and D.Zhang. A completed modeling of local binary pattern operator for texture classification. IEEE Transactions on Image Processing, 19(6):1657-1663, 2010. 